\title{
Appendices for A Fiedler Vector Scoring Approach for Novel RNA Motif Selection
}

\author{
Qiyao Zhu ${ }^{\dagger}$ and Tamar Schlick*,†, \\ $\dagger$ Courant Institute of Mathematical Sciences, New York University, New York, NY, 10012 \\ \$Department of Chemistry, New York University, New York, NY, 10003, and NYU-ECNU Center \\ for Computational Chemistry, NYU Shanghai, Shanghai 200062, P. R. China \\ E-mail: schlick@nyu.edu
}

\section{Appendix A: Details of Calcula- tions}

\section{A1. K-means}

The K-means algorithm is an unsupervised clustering method that divides a set of points into $K$ clusters. The initialization step randomly assigns $K$ centroids in the data domain. In each iteration, step 1 establishes $K$ clusters by grouping the points with their nearest centroids, and step 2 updates the $K$ centroids to be the means of points in the corresponding clusters. Steps 1 and 2 are repeated until the algorithm converges to an optimal solution, i.e., no change in cluster assignments of the points, or it reaches a maximum number of iterations.

\section{A2. k-nearest-neighbors (k-NN)}

$\mathrm{k}-\mathrm{NN}$ is a supervised classification algorithm that classifies a set of points into $K$ clusters. The algorithm requires $K$ training datasets with different labels, and a hyperparameter $k$, i.e., the number of neighbors. For each point, the algorithm checks the identities of the $k$ nearest neighbors in the training datasets, and label the point based on the identity of the majority of its neighbors.

\section{A3. Implementation in Matlab}

All computations are performed in Matlab (version R2019b). The linear and quadratic regression are performed using Matlab function linsolve() and polyfit() respectively. The PCA dimensionality reduction is performed using Matlab function $p c a()$. K-means clustering is performed using Matlab function kmeans(). The k-NN classification model is built using Matlab function fitcknn(). The cross-validation error of the model is calculated using kfoldLoss(), and model prediction is performed using predict(). Only a few seconds are required to run these functions using a MacBook Pro with $3.1 \mathrm{~Hz}$ Intel Core i5 processor and $8 \mathrm{~GB}$ RAM.

\section{Appendix B: Hall's Minimiza- tion using Fiedler Vectors}

Kenneth Hall in 1970 solved a minimization problem using eigenvectors of Laplacian matrices. ${ }^{1}$ Specifically, given $n$ connected points on a line positioned at $\left(x_{1}, x_{2}, \ldots, x_{n}\right)$ with adjacency matrix $A$ (without self-loops), he sought to position these points such that the weighted sum of squared distances, $z$

$$
z=\frac{1}{2} \sum_{i=1}^{n} \sum_{j=1}^{n}\left(x_{i}-x_{j}\right)^{2} a_{i j}
$$

is minimized, subject to

$$
\sum_{i=1}^{n} x_{i}^{2}=1
$$


Hall defines the row vector $X^{T}=\left(x_{1}, x_{2}, \ldots, x_{n}\right)$, and minimizes the quadratic form $z=X^{T} L X$ subject to $X^{T} X=1$, where $L$ is the $n$ by $n$ Laplacian matrix. The trivial minimum $z=0$ occurs at $X^{T}=(1,1, \ldots, 1) / \sqrt{n}$. Using Lagrange multipliers, the nontrivial minimum $z=\lambda_{2}$ is achieved at the solution $X^{T}=\mu_{2}$.

\section{Appendix C: Fiedler Vector Ob- servations, Fiedler Values and Compactness}

We apply Hall's minimization to explain our observations for the pattern in $\boldsymbol{\mu}_{\mathbf{2}}$ components shown in Figure 3, and to explain the relation between compactness and $\lambda_{2}$. If the $n$ vertices of a tree graph are positioned in a line at $\left(x_{1}, x_{2}, \ldots, x_{n}\right)$, so that the centroid of the graph is at 0 , i.e., $\sum_{i=1}^{n} x_{i}=0$, then Hall's solution translates into: setting $\left(x_{1}, x_{2}, \ldots, x_{n}\right)=\left(\mu_{2,1}, \mu_{2,2}, \ldots, \mu_{2, n}\right)$ minimizes

$$
\frac{\sum_{(i, j) \in E}\left(x_{i}-x_{j}\right)^{2}}{\sum_{i=1}^{n} x_{i}^{2}},
$$

where $E$ is the set of edges of the graph, and the minimum equals to $\lambda_{2}$. If we assume that $\lambda_{2}$ is simple, then $\mu_{2}$ is the unique nontrivial minimizer up to normalization.

We first interpret our Fiedler vector observations. Given the different modules that the vertices are in, there are intuitive ways that these vertices should be positioned so that (C.1) is minimized. Because each Fiedler vector component $\mu_{2, i}$ is associated with vertex $i$ and $\boldsymbol{\mu}_{\mathbf{2}}$ uniquely minimizes (C.1), the values of correponding $\mu_{2, i}$ should reflect these intuitive ways of vertex arrangement.

\section{Observation 1:}

The Fiedler vector components of linear modules are in ascending order.

\section{Explanation:}

Given a linear subgraph, increasing the distance between two vertices, while keeping the distance between two adjacent vertices unchanged increases the denominator $\sum_{i=1}^{n} x_{i}^{2}$, and thus minimizes (C.1). As $\boldsymbol{\mu}_{2}$ is the unique minimizer, the corresponding components are monotonically in- creasing.

\section{Observaion 2:}

The two free end branch vertices inside 3-way branched modules have same Fiedler vector components.

\section{Explanation:}

For two branch vertices positioned at $x_{b 1}$ and $x_{b 2}$, and center vertex positioned at $x_{C}$, the contributions to the numerator in (C.1) are $\left(x_{b 1}-x_{C}\right)^{2}$ and $\left(x_{b 2}-x_{C}\right)^{2}$, and to the denominator are $x_{b 1}^{2}$ and $x_{b 2}^{2}$. By uniqueness of the minimizer $\boldsymbol{\mu}_{\mathbf{2}}$, these branch vertices should be assigned the same Fiedler vector component values.

\section{Observaion 3:}

Inside 3-way branched modules, if the free end branch vertices precede the center vertex, their Fiedler vector components are smaller than that of the center vertex; otherwise, larger.

\section{Explanation:}

Using same notations as Observation 2, for a free branch vertex preceding the center vertex, we have $x_{C}<0$. If $\left(x_{b 1}-x_{C}\right)^{2}$ is fixed for the numerator, the denominator contribution is $x_{b 1}^{2}=\left[\left(x_{b 1}-x_{C}\right)+\right.$ $\left.x_{C}\right]^{2}$. Thus, to maximize the denominator, $x_{b 1}-$ $x_{C}<0$, or $x_{b 1}=x_{b 2}<x_{C}$.

Next, we give an intuitive explanation of why compact tree graphs have larger Fiedler values. We know $\lambda_{2}$ minimizes (C.1) with $\mu_{2}$ being the minimizer, so

$$
\lambda_{2}=\frac{\sum_{(i, j) \in E}\left(\mu_{2, i}-\mu_{2, j}\right)^{2}}{\sum_{i=1}^{n} \mu_{2, i}^{2}} .
$$

Consider the two tree graphs 7_1 and 7_2 shown below. At left, the vertices are numbered from 1 to 7 , and the Fiedler values are given. At right, the Fiedler vector components are mapped onto their corresponding vertices.

In both graphs, vertices 3-7 are in linear modules, and their corresponding $\mu_{2, i}$ values monotonically increase. In 7_1, vertex 1 is adjacent to 2 , and 2 to 3 , so they contribute $\left(\mu_{2,2}-\mu_{2,3}\right)^{2}+$ $\left(\mu_{2,1}-\mu_{2,2}\right)^{2}$ to the numerator, and $\mu_{2,2}^{2}+\mu_{2,1}^{2}=$ 
$\left(\left|\mu_{2,3}\right|+\left|\mu_{2,2}-\mu_{2,3}\right|\right)^{2}+\left(\left|\mu_{2,3}\right|+\left|\mu_{2,2}-\mu_{2,3}\right|+\right.$ $\left.\left|\mu_{2,1}-\mu_{2,2}\right|\right)^{2}$ to the denominator. However, in 7_2, vertices 1 and 2 are both adjacent to 3, and $\mu_{2,1}=\mu_{2,2}$. As a result, their contribution to the numerator is the same, but their contribution to the denominator becomes $\mu_{2,2}^{2}+\mu_{2,1}^{2}=\left(\left|\mu_{2,3}\right|+\right.$ $\left.\left|\mu_{2,2}-\mu_{2,3}\right|\right)^{2}+\left(\left|\mu_{2,3}\right|+\left|\mu_{2,2}-\mu_{2,3}\right|\right)^{2}$.

We see that whenever there is a branched module, the free end branch vertices contribute less to the denominator of (C.2) than if they were in a linear module. Consequently, $\lambda_{2}$ will be larger. Hence, compact tree graphs have larger Fiedler values.

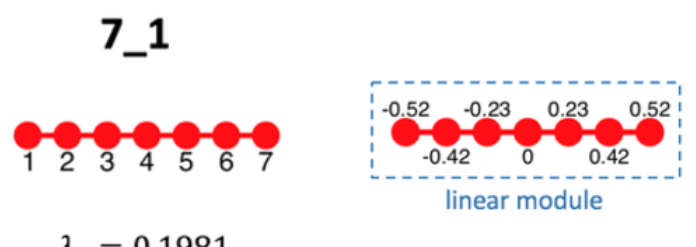

$\lambda_{2}=0.1981$
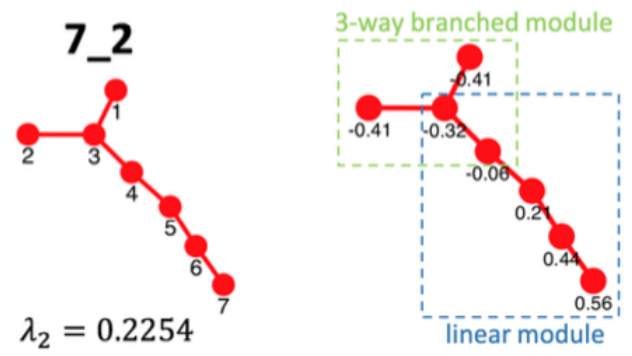

\section{Appendix D: Slope $s$ Derivation}

The points we want to perform least squares regression are $\left(1, \tilde{v_{1}}\right),\left(2, \tilde{v_{2}}\right), \ldots,\left(n, \tilde{v_{n}}\right)$. In the linear regression, the line is $y=s x+b$, where $s$ is the slope, and $b$ is the $y$-intercept. Let us denote the fitted values at $x=i$ for $i=1,2, \ldots, n$ as $y_{i}$, i.e., $y_{i}=s i+b$. Then $s$ and $b$ minimize

$$
R=\sum_{i=1}^{n}\left(y_{i}-\tilde{v}_{i}\right)^{2}=\sum_{i=1}^{n}\left(s i+b-\tilde{v}_{i}\right)^{2} .
$$

Hence, the gradient of $R$ with respect to $s$ and $b$ should be 0 , i.e.,

$$
\frac{\partial R}{\partial s}=\sum_{i=1}^{n} 2 i\left(s i+b-\tilde{v_{i}}\right)=0
$$

$$
\frac{\partial R}{\partial b}=\sum_{i=1}^{n} 2\left(s i+b-\tilde{v}_{i}\right)=0
$$

We simplify (D.2) to express $b$ in terms of $s$. Recall that the Fiedler vector components sum up to 0 , so $\sum_{i=1}^{n} \tilde{v}_{i}=0$. Thus,

$$
b=-\frac{1}{n} \sum_{i=1}^{n} s i=-s \cdot \frac{n+1}{2} .
$$

We substitute this back to (D.1) to obtain

$$
\sum_{i=1}^{n} i\left(s i-s \cdot \frac{n+1}{2}-\tilde{v}_{i}\right)=0 .
$$

Organizing the terms,

$$
\begin{gathered}
s \sum_{i=1}^{n} i^{2}-s \frac{n+1}{2} \sum_{i=1}^{n} i-\sum_{i=1}^{n} i \tilde{v}_{i}=0, \\
s \cdot \frac{n(n+1)(2 n+1)}{6}-s \cdot \frac{n+1}{2} \cdot \frac{n(n+1)}{2}-\sum_{i=1}^{n} i \tilde{v}_{i}=0 .
\end{gathered}
$$

Hence, we see that

$$
s=\frac{12}{(n-1) n(n+1)} \sum_{i=1}^{n} i \tilde{v}_{i} .
$$

\section{References}

(1) Hall, K. M. An r-dimensional quadratic placement algorithm. Management Science 1970, 17, 219-229. 